\title{
REDUCING CRASH RISK IN VISUALLY IMPAIRED OLDER DRIVERS: MEDICAL-SURGICAL VERSUS EDUCATIONAL INTERVENTIONS
}

\author{
Gerald McGwin, Jr. and Cynthia Owsley \\ University of Alabama, Birmingham \\ Department of Ophthalmology \\ 700 18th Street South, Suite 609 \\ Birmingham, AL 35294 USA \\ E-mail: mcgwin@uab.edu
}

\begin{abstract}
\section{OBJECTIVES}

The aim of this paper is to compare the effectiveness of a medical-surgical intervention and an educational intervention in reducing the rate of crash involvement among visually impaired older drivers. Visual impairment is a common functional problem in older adults. Older drivers with visual processing deficits have an increased crash risk. Interventions that reduce these risks need to be identified in order to enhance driver safety.
\end{abstract}

\section{METHODS}

We have conducted two studies in an effort to examine two types of interventions to lower crash risk in visually impaired drivers. In a prospective cohort study we focused on 277 older drivers with cataracts, about half of who elected surgery and intraocular lens implantation at baseline, and the other half who declined surgery. They were followed for police-reported crash involvement for four to six years. In a second study, 403 older drivers who were visually impaired (acuity and/or useful field of view deficit) were randomly assigned to an individually administered and tailored educational intervention plus usual care, or to usual-care-only. The educational intervention promoted the use of self-regulatory driving strategies and was based on current models of health behavior change. Usual care was a comprehensive eye exam. In this study, subjects were also followed for police-reported crash involvement.

\section{RESULTS}

With respect to the study evaluating the cataract surgery intervention, patients who underwent cataract surgery had half the rate of crash involvement during follow-up compared with cataract patients who did not undergo surgery (rate ratio [RR] $0.47,95 \%$ confidence interval [CI] 0.23 to 0.94; $\mathrm{p}<0.05$ ). With respect to the study evaluating the educational intervention, those receiving the intervention were similar to the usual-care-only group in terms of their crash involvement rate (RR $1.40,95 \%$ CI 0.92 to $2.12 ; \mathrm{p}>0.05$ ). 


\section{CONCLUSIONS}

Cataract surgery has a previously undocumented benefit for older driver safety. However, an individualized educational intervention to promote safe driving strategies did not enhance driver safety. The most effective public health initiatives for reducing crash risk in older drivers may be to focus on the timely treatment of chronic medical conditions in order to prevent, reverse, or slow functional decline. Evidence that educational programs improve older driver safety remains unavailable. 